\title{
Analyzing and Visualizing the Impact of Covid- 19 on the World
}

\author{
*Mr.Omkar Shelar, \#Dr. Jyoti Kharade \\ *Student, \#Associate Professor, Bharati Vidyapeeth's Institute of Management and Information \\ Technology, Navi Mumbai, India. *shelaromkar10@gmail.com , \# kharadejyoti09@gmail.com
}

Abstract - The Novel Corona virus is emerging as a Global public health threat. The outbreak initially emerged in Wuhan, China, large numbers of patients were getting sick because of Pneumonia and later it was found that they were infected with the Novel Corona virus this emphasizes the importance of analyzing the data of this virus and predicting their risks of infecting people all around the globe. In this study, we present an effort to compile and analyze the outbreak information on COVID19 based on the open datasets on 2019 nCoV provided by the Johns Hopkins University, World Health Organization. An exploratory data analysis with visualizations has been made to understand the number of different cases reported (confirmed, death, and re-covered) in the World. Overall, at the outset of an outbreak like this, it is highly important to readily provide information to begin the evaluation necessary to understand the risks and begin containment activities.

Keywords - Data Visualization, Covid-19, Data Analysis, Dashboard, Novel Corona-Virus, Power Bi, SARS-COV-2

\section{INTRODUCTION}

The Novel Corona Virus is spreading rapidly in the major countries of the world and as no vaccine is been developed, the contagion is spreading day by day. To tackle the crisis many countries are on temporal lockdown. A global lockdown of this magnitude is affecting the world economy. So, paper presents an effort to compile and analyze the outbreak information on covid19, the data set is taken for from "center for systems science and engineering at Johns Hopkins University (JHU)".

There is an obvious concern globally regarding the fact about the emerging coronavirus 2019 novel coronavirus (2019- nCoV) as a worldwide public health threat. As the out-break of COVID- 19 causes by the severe acute respiratory syndrome coronavirus 2(SARS- CoV- 2) progresses within China and beyond, rapidly available epidemiological data are needed to guide strategies for situational awareness and intervention. The recent outbreak of pneumonia in Wuhan, China, caused by the SARS- CoV- 2 emphasizes the importance of analyzing the epidemiological data of this novel virus and predicting their risks of infecting people all around the globe. In this study, we present an effort to compile and analyze epidemiologica 1 outbreak information on COVID- 19 based on the several open datasets on 2019- nCoV provided by the Johns Hopkins University, World Health Organization, Chinese Center for Disease Control and Prevention, National Health Commission, and DXY. An exploratory data analysis with visualizations has been made to understand the number of different cases reported (confirmed, death, and recovered) in different provinces of China and outside of China. Overall, at the outset of an outbreak like this, it is highly important to readily provide information to begin the evaluation necessary to understand the risks and begin containment activities. There is an obvious concern globally regarding the fact about the emerging coronavirus2019 novel coronavirus (2019-nCoV) as a worldwide public health threat. As the out-break of COVID-19 causes by the severe acute respiratory syndrome coronavirus 2(SARS-CoV-2) progresses within China and beyond, rapidly available epidemiological data are needed to guide strategies for situational awareness and intervention. The recent outbreak of pneumonia in Wuhan, China, caused by the SARS-CoV-2 emphasizes the importance of analyzing the epidemiological data of this novel virus and predicting their risks of infecting people all around the globe. In this study, we present an effort to compile and analyze epidemiologica 1 outbreak information on COVID-19 based on the several open datasets on 2019-nCoV provided by the Johns Hopkins University, World Health Organization, Chinese Center for Disease Control and Prevention, National Health Commission, and DXY. An exploratory data analysis with visualizations has been made to understand the number of different cases reported (confirmed, death, and re-covered) in different provinces of China and outside of China. Overall, at the outset of an out break like this, it is highly important to readily provide information to begin the evaluation necessary to understand the risks and begin containment activities. 
There is an obvious concern globally regarding the fact about the emerging coronavirus 2019 novel coronavirus $(2019 \mathrm{nCoV})$ as a worldwide public health threat. As the outbreak of COVID19 causes by the severe acute respiratory syndrome coronavirus 2 (SARSCoV2) progresses within China and beyond, rapidly available epidemiological data are needed to guide strategies for situational awareness and intervention. The recent outbreak of pneumonia in Wuhan, China, caused by the SARS- CoV- 2 emphasizes the importance of analyzing the epidemiological data of this novel virus and predicting their risks of infecting people all around the globe. In this study, we present an effort to compile and analyze epidemiologica 1 outbreak information on COVID- 19 based on the several open datasets on 2019- nCoV provided by the Johns Hopkins University, World Health Organization, Chinese Center for Disease Control and Prevention, National Health Commission, and DXY. An exploratory data analysis with visualizations has been made to understand the number of different cases reported (confirmed, death, and recovered) in different provinces of China and outside of China. Overall, at the outset of an outbreak like this, it is highly important to readily provide information to begin the evaluation necessary to understand the risks and begin containment activities.

\section{The objectives of research paper are:}

1. To analyze the spread of the novel corona-virus on the world.

2. Visualizing the impact of Covid-19.

3. To find confirmed cases, death totals, recovered cases, non-recovered cases, recovery rate and active cases.

\section{Literature REVIEW}

Samrat K.Dey [1] helps the reviewer with Data Analysis and Exploratory data analysis. In his paper he visualizes the data and fights trends and insight on the Data.

Jrhau Lung, [2] help reviewers with the chemical structure of the anti-Sars-Cov-2 RNA-dependent RNA polymerase. This paper shows us the actual RNA of the Virus and the components of the Virus.

Richard I. Horowitz [3] publish a paper on the Efficacy of Glutathione Therapy in Relieving Dyspnea Associated With COVID-19 Pneumonia: A Report of 2 Cases. In his paper he species the Glutathione Therapy in Relieving Dyspnea Associated With COVID-19 Pneumonia.

Zhu N, Zhang [4] in this paper the author reviews the Virus Genome Structure and the test the Various results of Covid19 Pneumonia patients according to their age.
Drosten .C [5] in his paper the author writes about the transmission, Scientific Structure and Diagnosis about the virus.

Lauren G. [6] this is a Dashboard published by CSSE at John Hopkins University (JHU) this dashboard contains live information about the world wide Active cases, Deaths, Recovered patients, etc.

Centers for Disease Control and Prevention.[7] here we can see the Cases Count in United states according to the Race of the Individuals, age, new cases day by day, etc.

Ji Wie [8] studies the Cross-species transmission of the newly identified coronavirus 2019-nCoV. This paper studies that how the Virus can be Spread from one species to another.

\section{RESEARCH METHODS}

Open dataset of $2019 \mathrm{nCoV}$ provided by the Johns Hopkins University is used for the research paper. An exceptional dashboard using the affected cases data to date, provided the data available in Google sheets format. This data-set consists of day to day information on the number of affected cases, deaths, and recovery on the cases of novel coronavirus.

Power BI is a collection of software services, apps, and connectors that work together to turn our unrelated sources of data into coherent, visually immersive, and interactive insights. It is one of the Powerful Data Analytics and Visualization tool.

The first step is to load the Dataset in power BI and then transform it according to requirements and Model the dataset to create a relationship between the individual tables so that the data can be accessed from one individual table to another. After data modeling in the next step is to create charts, metrics, tables and visuals to get insight on the Data. We would be creating tables, Charts, maps in power Bi to analyze and Visualize the Spread of the Virus.

\section{DATA ANALYSIS \& VISUALIZATION}

The dataset is analyzed with different EDA methods and is visualize data to provide a sufficient consciousness regarding the outbreak of COVID 19 all over the globe. Research paper analyze and visualize data between 22 January 2020 and 9 April 2020. However, at first massive number of cases were reported from China compared to the rest of the world, and then the Pandemic started spreading to other countries, the most affected countries after china are United States, Spain, Italy, France, Germany, Iran, United Kingdom, etc. 
Figure 1: Visual Demonstration

\begin{tabular}{|c|c|c|c|c|}
\hline Country & Confirmed & 3 Day Change & Recovery Rate & Deaths \\
\hline United States & $4,61,437$ & $26 \%$ & $5.51 \%$ & 16,478 \\
\hline Spain & $1,53,222$ & $12 \%$ & $34.05 \%$ & 15,447 \\
\hline Italy & $1,43,626$ & $8 \%$ & $19.82 \%$ & 18,279 \\
\hline France & $1,18,781$ & $20 \%$ & $19.71 \%$ & 12,228 \\
\hline Germany & $1,18,181$ & $14 \%$ & $44.34 \%$ & 2,607 \\
\hline China & 82,883 & $0 \%$ & $93.72 \%$ & 3,339 \\
\hline Iran & 66,220 & $9 \%$ & $48.79 \%$ & 4,110 \\
\hline United Kingdom & 65,872 & $26 \%$ & $0.54 \%$ & 7993 \\
\hline Turkey & 42,282 & $40 \%$ & $5.07 \%$ & 908 \\
\hline Belgium & 24,983 & $20 \%$ & $20.67 \%$ & 2,523 \\
\hline Switzerland & 24,051 & $11 \%$ & $44.07 \%$ & 948 \\
\hline Netherlands & 21,903 & $16 \%$ & $1.27 \%$ & 2,403 \\
\hline Canada & 20,654 & $25 \%$ & $24.99 \%$ & 503 \\
\hline Brazil & 18,092 & $49 \%$ & $0.96 \%$ & 950 \\
\hline Portugal & 13,956 & $19 \%$ & $1.47 \%$ & 409 \\
\hline Austria & 13,244 & $8 \%$ & $39.57 \%$ & 295 \\
\hline South Korea & 10,423 & $1 \%$ & $66.90 \%$ & 204 \\
\hline Russia & 10,131 & $60 \%$ & $6.89 \%$ & 76 \\
\hline Israel & 9,968 & $12 \%$ & $10.14 \%$ & 86 \\
\hline Sweden & 9,141 & $27 \%$ & $2.24 \%$ & 793 \\
\hline India & 6,725 & $41 \%$ & $9.22 \%$ & 226 \\
\hline Ireland & 6,574 & $23 \%$ & $0.38 \%$ & 263 \\
\hline Norway & 6,211 & $6 \%$ & $0.52 \%$ & 108 \\
\hline Australia & 6,108 & $5 \%$ & $24.10 \%$ & 51 \\
\hline Chile & 5,972 & $24 \%$ & $21.33 \%$ & 57 \\
\hline Denmark & 5,830 & $20 \%$ & $32.30 \%$ & 237 \\
\hline Poland & 5,575 & $26 \%$ & $5.09 \%$ & 174 \\
\hline Czech Republic & 5,569 & $15 \%$ & $5.40 \%$ & 112 \\
\hline Peru & 5,256 & $105 \%$ & $27.36 \%$ & 138 \\
\hline Romania & 5,202 & $28 \%$ & $12.44 \%$ & 248 \\
\hline Ecuiador & 1065 & $22 \%$ & 68204 & 272 \\
\hline Total & $15,95,350$ & $19 \%$ & $22.19 \%$ & 95,455 \\
\hline
\end{tabular}

Figure 1 depicts the visual demonstrating the confirmed cases according to the country, 3 days change, recovery rate and deaths.

As on $9^{\text {th }}$ April 2020 the number of confirmed cases in united states were $4,61,437,3$ day change was $26 \%$, recovery rate was $5.51 \%$ and total deaths were 16,478 . Similarly, the number of confirmed cases in Spain were $1,53,222,3$ day change was $12 \%$ and recovery rate was $34.05 \%$ and total deaths were 15,447 . There is a huge gap in the numbers of confirmed cases for both of these countries but there is less gap in their death counts. Similarly, in Italy the confirmed cases are less than Spain and United States but their death count is highest in the world.

The study is to analyze three different categories of data including confirmed cases, death, and recovered cases in the world from a time period of 22 January to 9 April 2020. This will provide a comparative analysis of all the cases reported in the world. After analyzing, there were 15,95,350 confirmed cases of COVID 19 on $9^{\text {th }}$ April 2020. The total countries affected are shown in figure 2. Figure 3 shows the severity of the pandemic.

The Figure 2 denotes the Spread of Novel Corona virus on the Global Map it shows the Spread of the virus and it's Severity on different countries as we can see that North America is More affected than South America and there are more number of Cases in North America as compared to South America. According to the figure the most Affected countries in the World were European Countries. As we can see that Australia, Africa and Asia have less number of known Cases but the Spread is not as Severe as Compared to U.S.A.

Figure 2: Spread of Novel Corona Virus

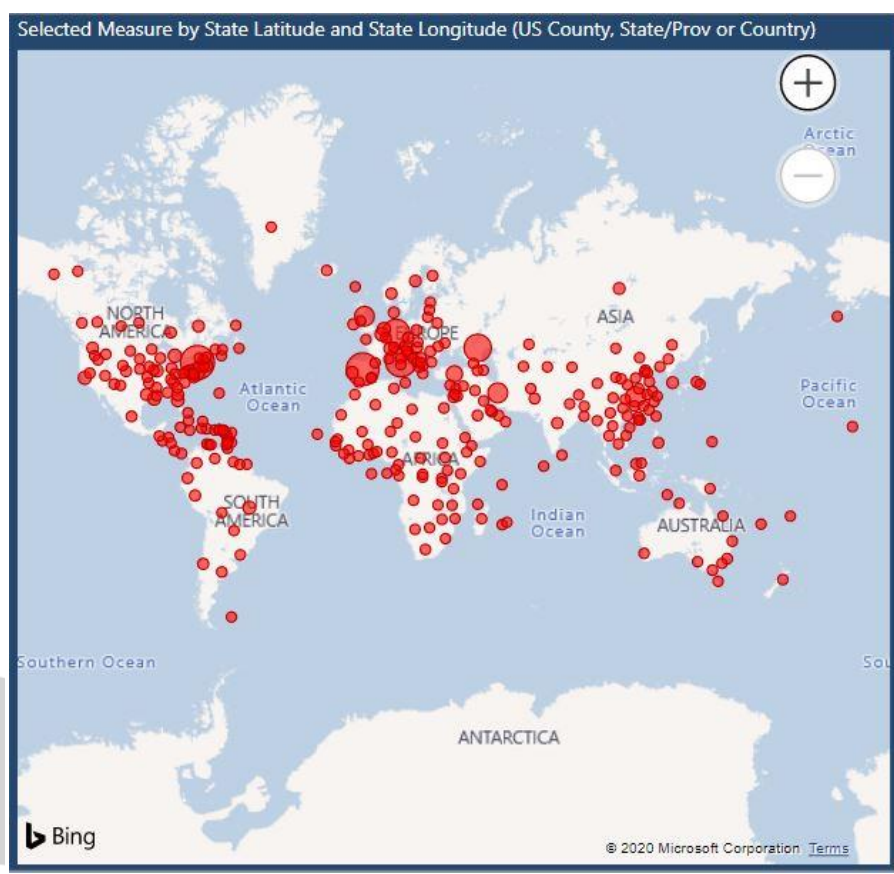

Figure 3: Severity of the pandemic

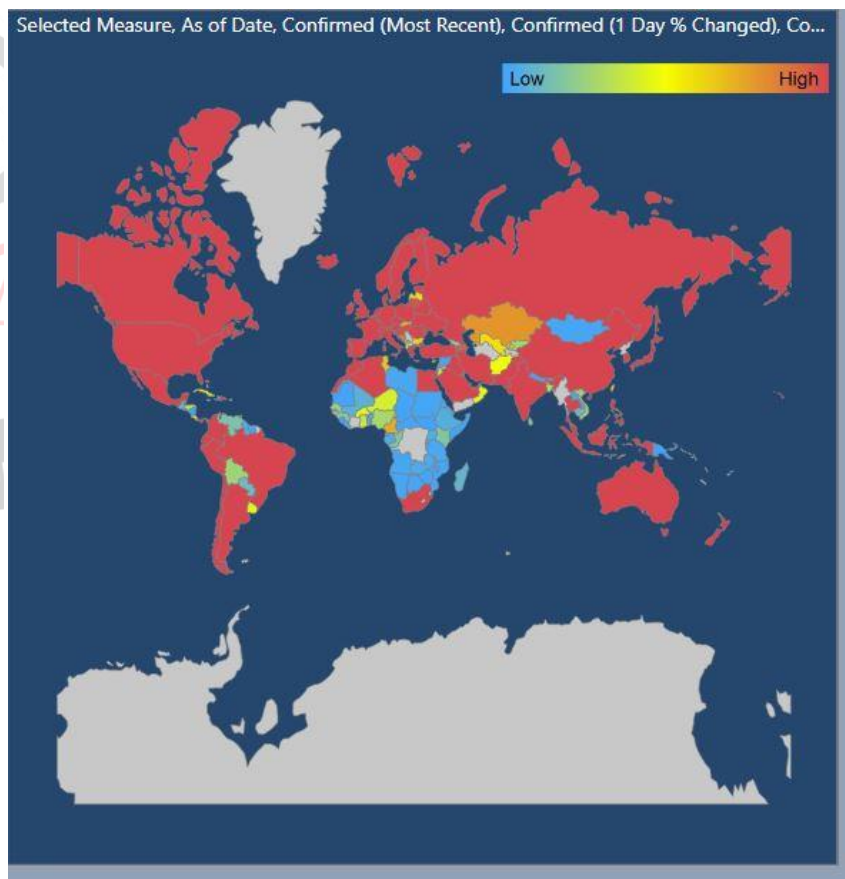

In Figure 3 we can see the Severity of the spread of Novel Corona Virus on the world map, the countries Colored red are the Countries which are most affected by the Spread of the virus and countries which are colored white have no known cases of novel corona virus and countries which are colored apart from red and white have less severity of spread. 
Figure 4: Recovery Rate

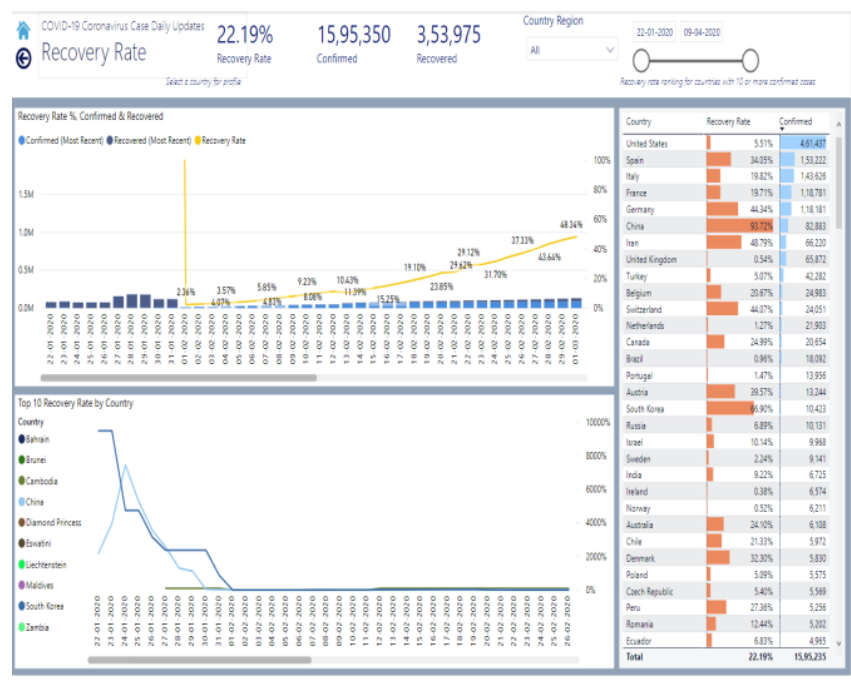

Figure 4 shows the recovery rate as we can see from 01-022020 to $01-03-2020$ the recovery rate has gone up from $2.36 \%$ to $48.34 \%$ and the global recovery rate is $22.19 \%$ as $15,95,350$ confirmed cases globally out of which $3,53,975$ have recovered. The most affected Country is United States as the total Case Count reached to $4,61,437$ followed by Spain-1,53,222, Italy-1,43,626, France- 1,18,781, Germany$1,18,181$, China- 82,883 and so on.

\section{RESULTS}

The Global Cases Count as of $9^{\text {th }}$ April 2020 are as follows:

- Confirmed Cases- 15,95,350

- Active Cases- 12,41,375

- Recovered Cases- 3,53,975

- Non-Recovered Cases- 12,41,375

- Recovery Rate- $22.19 \%$

- Deaths Worldwide- 95,455

Total Covid-19 cases Worldwide were analyzed and the visuals indicate that the most affected Countries in the world are USA, Spain, Italy, France, Germany, China, Iran, UK, Turkey. More than 187 countries and territories in the world are affected by Novel Coronavirus and the Spread is increasing day by day.

\section{CONCLUSION}

It is found that as on $9^{\text {th }}$ April 2020 United States is leading in number of confirmed cases in the world rising to $4,61,437$ and having a deaths total to 16,478 and $4,36,027$ total non-recovered cases.

The most populated country in the world where the outbreak began that is China has the highest recovery rate in the world ranging to $93.72 \%$ no other countries have such a high rate of recovery and the confirmed cases count is just 82,883 and death count is 3,339 .
In Italy the confirmed cases are 1,434,626 less than US and Spain but the death count is highest in the world ranging to 18,279 .

The visuals indicate that the most affected Countries in the world are USA, Spain, Italy, France, Germany, China, Iran, UK, Turkey.

In UK confirmed cases were 65,872 and active cases were 65,513 and no of deaths 7,993 but the recovery rate is $0.54 \%$ which is very less as compared to other affected countries.

The pandemic of such a magnitude can be handled but we need correct data to analyze the patterns or the trends in the data, so with the help of this findings Governments of the world can take measures and develop strategies to stop the spread of the Virus.

\section{REFERENCES}

[1] Samrat K.Dey, . Md. Mahbubar Rahman, Umme R.Siddiqi, Arpita Howlader, Analyzing the epidemiological outbreak of COVID 19: A visual exploratory data analysis approach. https://doi.org/10.1002/jmv.25743

[2] Jrhau Lung, YuShih Lin, YaoHsu Yang, YuLun Chou, LiHsin Shu, YuChing Cheng, Hung Te Liu and ChingYuan Wu, The potential chemical structure of antiSARSCoV2 RNAdependent RNA polymerase, Journal of Medical Virology,92, 6, (693-697), (2020). https://publons.com/publon/30792362/

[3] Richard I. Horowitz, Phyllis R. Freeman and James Bruzzese, Efficacy of glutathione therapy in relieving dyspnea associated with COVID-19 pneumonia: A report of 2 cases, Respiratory MedicineCaseReports, https://www.ncbi.nlm.nih.gov/pubme $\mathrm{d} / 32322478$

[4] Zhu N, Zhang D, Wang W, et al. A novel coronavirus from patients with pneumonia in China,2019. N.Engl,J,Med. 2020; 382: 727- 733. https://doi.org/10.1056/NEJMoa2001017

[5] Drosten C, Günther S, Preiser W, et al. Identification of a novel coronavirus associated with severe acute respiratory syndrome. N Engl J Med. 2003; 348: 19671976.

[6] Lauren G. Coronavirus COVID19 Global Cases by Johns Hopkins CSSE January 23, 2020. https://gisanddata.maps.arcgis.com/apps/opsdashboard /index.html.

[7] Centers for Disease Control and Prevention. 2019 Novel Coronavirus (2019-nCoV), Wuhan, China. 2019. https://www.cdc.gov/coronavirus/2019-nCoV/summar y.html

[8] Ji Wie, Wang W, Zhao X, Zai J, Li X. Cross-species transmission of the newly identified coronavirus 2019-nCoV. $J \quad M e d$ Virol. 2020; 92: 433 440. https://doi.org/10.1002/jmv.25682 